\title{
Bone Safety Profile of Steroidal Aromatase Inhibitor in Comparison to Nonsteroidal Aromatase Inhibitors in Postmenopausal Women with Breast Cancer: A Network Meta-Analysis
}

\author{
Shanshan Chen Lan Bo Dan Lv Fei Ma \\ Department of Medical Oncology, National Cancer Center/National Clinical Research Center for Cancer/Cancer \\ Hospital, Chinese Academy of Medical Sciences and Peking Union Medical College, Beijing, China
}

\section{Keywords}

Bone $\cdot$ Network · Meta-analysis · Aromatase inhibitor . Tamoxifen $\cdot$ Exemestane

\begin{abstract}
Background and Objectives: Aromatase inhibitors (Als) provide an alternative to tamoxifen as an adjuvant therapy for postmenopausal patients with breast cancer (BC). Large trials resulted better outcomes with Als. Adjuvant therapy with Als reduced the risk of relapse compared with tamoxifen. Systemic therapies for BC can interfere with bone turnover, either by affecting gonadal steroid hormone production or by inhibiting peripheral aromatization into estrogen. We aimed to evaluate the safety profile of bone-related events by comparing 3 Als with tamoxifen and a placebo. Methods: The Preferred Reporting Items for Systematic Reviews and Meta-Analysis guidelines were used for network meta-analyses (NMAs). Searches were performed using PubMed, Embase/Medline, Cochrane, and Ovid databases. Randomized controlled trials comparing tamoxifen and placebo or other Als to steroidal or nonsteroidal Als in patients with $B C$ reporting bone-related safety events were included in NMA. NMA in a Bayesian approach was performed using $R$ software (ver 3.2), Gemtc package. Results: Seventeen studies reporting 4 different bone-related endpoints were included. Although there was no statistical significance, treatment with exemestane lowered the incidence of bone pain (odds ratio [OR] vs. anastrozole and letrozole: $0.63,0.54$ ), fracture episodes (OR vs. anastrozole and letrozole: $0.84,0.80)$, and osteoporosis (OR vs. anastrozole and letrozole: $0.85,0.73$ ) compared with
\end{abstract}

Karger@karger.com www.karger.com/brc

Karger $\%$

马OPEN ACCESS
(C) 2022 The Author(s)

Published by S. Karger AG, Basel

This is an Open Access article licensed under the Creative Common Attribution-NonCommercial-4.0 International License (CC BY-NC) (http://www.karger.com/Services/OpenAccessLicense), applicable to the online version of the article only. Usage and distribution for commercial purposes requires written permission. letrozole and anastrozole. Reduction in bone mineral density was lesser in exemestane than in anastrozole (mean reduction in hip: 1.05; lumbar spine: 1.25). Treatment ranking with the surface under the cumulative ranking curve showed that exemestane was found to reduce the incidence of bonerelated adverse events. Conclusion: A lower incidence of bone-related safety events was observed in patients treated with exemestane.

(c) 2022 The Author(s). Published by S. Karger AG, Basel

\section{Introduction}

Breast cancer (BC) is the prevalent cause of malignancy with an incidence of 2.26 million new cases [1]. Estrogen receptors (ERs) are nuclear proteins that regulate the expression of specific genes, and approximately $80 \%$ of BCs express ER [2]. In previous trials [3], aromatase inhibitors (AIs) have shown to be more effective than selective ER modulator (SERM), such as tamoxifen in neoadjuvant, adjuvant, and metastatic settings [4-7]. AIs are used as adjuvant therapy in $\mathrm{ER}+\mathrm{BC}$ as per the National Comprehensive Cancer Network (NCCN) and the European Society for Medical Oncology (ESMO) guidelines $[8,9]$. AIs are used for a longer duration, and continuous deprivation of estrogen may affect other physiological functions, which leads to lower adherence [10]. Coupled with increased survival, relative risks of age-associated diseases, and interruption of physiological functions of estrogen, musculoskeletal changes are reported in patients treated with AIs in adjuvant settings [11]. 
Estradiol is important for the development and progression of BC in women with ER + early BC [12]. Endocrine therapy prevents deleterious effects of estradiol in breast tissue by depleting estradiol concentration or by preventing its local action in breast tissue (SERMs, i.e., tamoxifen), thereby improving oncological outcomes [13]. The use of AIs in postmenopausal women leads to systemic estradiol depletion, which affects the overall skeletal health [14]. Estradiol-deficient state causes increased bone remodeling and negative bone balance. This results in bone loss, microstructural deterioration, and bone fragility predisposing to fractures [12, 15]. Similar effects are observed with tamoxifen in premenopausal women $[12,16]$. In contrast, the use of tamoxifen in postmenopausal women appears to exert its protective effects on bone, but studies on fracture risk are inconclusive [12, 17]. The longevity of women with ER $+B C$ treated who received adjuvant endocrine therapy emphasizes the need to mitigate adverse skeletal effects of these therapies to maximize benefit. Fractures are associated with increased morbidity, mortality, and a high socioeconomic burden $[15,18]$.

Two types of AIs are available: a steroidal, irreversible aromatase inactivator exemestane [19] and a nonsteroidal reversible imidazole (anastrozole [20] and letrozole [21]). Exemestane is a third-generation AI [22]. It was approved for use in postmenopausal patients with metastatic BC after progression with tamoxifen [22]. Its efficacy was proven in the adjuvant setting and was routinely used $[23,24]$. With respect to relative efficacies of different AIs, a recent meta-analysis reported similar efficacy [25]. With exemestane, the incidence of bonerelated adverse events (AEs) may be comparatively less in patients treated in the adjuvant settings than the other 2 AIs. Clinical trials reporting bone-related events did not have sufficient statistical power for providing effect estimates on the incidence of bone-related events. A recent randomized, phase 3 trial conducted on 4,136 postmenopausal patients with hormone receptor-positive and node-positive early BC showed noninferiority of letrozole to anastrozole in terms of the endpoints such as disease-free survival, overall survival, and safety. However, the study was terminated prematurely due to lower than expected disease-free survival events. Moreover, this study did not account for any bone-related events [26]. Furthermore, a meta-analysis comparing the safety of all third-generation AIs focused on bone health is limited. Hence, limited clinical data present a challenge to optimize disease management. The goal of this mixed treatment comparison network meta-analyses (NMAs) is to compare the relative safety of exemestane with letrozole and anastrozole in terms of the incidence of bone-related AEs.

\section{Materials and Methods}

Identification and Development of Search Strings

A comprehensive systematic literature review was performed according to the prespecified protocol after the Preferred Reporting Items for Systematic Reviews and Meta-Analysis (PRISMA) recommendations for systematic reviews incorporating NMA [26]. The PRISMA-NMA checklist of items was used when reporting a systematic review. Electronic medical databases (PubMed, Embase/Medline, Cochrane Library, and Ovid database) were searched with search strategy formulated using the Patient, Intervention, Comparator, Outcome, Study type methodology. RCTs in patients with BC treated with AIs in adjuvant settings compared with tamoxifen, placebo, or other AIs reporting bone safety events were considered (shown in Fig. 1).

Literature was searched since its inception through March 12, 2021 with the following search string: ("breast tumor"/exp OR "breast gland tumor" OR "breast gland tumour" OR "breast mass" OR "breast neoplasms" OR "breast neoplasms, male" OR "breast tumor" OR "breast tumour" OR "female breast neoplasm" OR "female breast tumor" OR "female breast tumour" OR "mamma tumor" OR "mamma tumour" OR "mammary gland tumor" OR "mammary gland tumour" OR "mammary neoplasms" OR "mammary tumor" OR "mammary tumor cell" OR "mammary tumour" OR "mammary tumour cell" OR "unilateral breast neoplasms") AND ("letrozole"/exp OR "1 (4, 4' dicyanobenzhydryl) 1, 2, 4 triazole" OR " $4,4^{\prime}$ ( 1 h 1, 2, 4 triazol 1 ylmethylene) bis (benzonitrile)" OR "cgs 20267" OR "cgs20267" OR "femar" OR "femara" OR "letrozole" OR "loxifan" OR "anastrozole"/exp OR " $2,2^{\prime}$ [5 (1 h 1, 2, 4 triazol 1 ylmethyl) 1, 3 phenylene] bis (2 methylpropionitrile)" OR "anastrozole" OR "anastrozole" OR "arimidex" OR "ici d1033” OR “icid1033" OR "trozolet” OR “zd 1033” OR “zd1033” OR "exemestane"/exp OR "6 methyleneandrosta 1, 4 diene 3, 17 dione" OR "aromasin" OR "aromasine" OR "exemestane" OR "fce 24304" OR "fce24304” OR "nakides” OR "nikidess" OR "pnu 155971” OR "pnu155971”) AND ("risk assessment”/exp OR "assessment, safety" OR "risk adjustment" OR "risk analysis" OR "risk assessment” OR "risk evaluation" OR "safety assessment" OR "osteoporosis"/exp OR "decalcification, pathologic" OR "endocrine osteoporosis" OR "osteoporosis" OR "osteoporotic decalcification" OR "fracture"/exp OR "bone cement fracture" OR "bone fracture" OR "closed fracture" OR "fracture" OR "fractures" OR "fractures, bone" OR "fractures, closed" OR "skeleton fracture" OR "unstable fracture") AND (placebo). Retrieved hits were compiled in a master sheet in Excel, duplicates were removed, a consolidated list of studies was screened for inclusion based on title, abstract, and full text of the publications.

\section{Inclusion/Exclusion Criteria}

Studies reporting bone safety endpoints of RCTs that compare AIs with tamoxifen, placebo-controlled studies, or other AIs in adjuvant and early BC settings were included. In case of multiple studies reporting the results of the same RCT, updated studies with relevant endpoints were included. Studies reporting other outcomes/endpoints and studies in neoadjuvant and advanced/metastatic BC settings along with non-English articles were excluded.

\section{Screening of Articles}

Two reviewers initially screened the titles and abstracts (where available) of all identified studies, according to predefined inclusion and exclusion criteria. If disagreements arose, discussions were conducted until a consensus was reached or a third reviewer was consulted. Full-text reviews and evaluations were obtained for articles and data extraction was then performed using a prespecified data-extraction template in Microsoft Excel. One researcher extracted the data, while another researcher and a statistician independently verified its accuracy. 


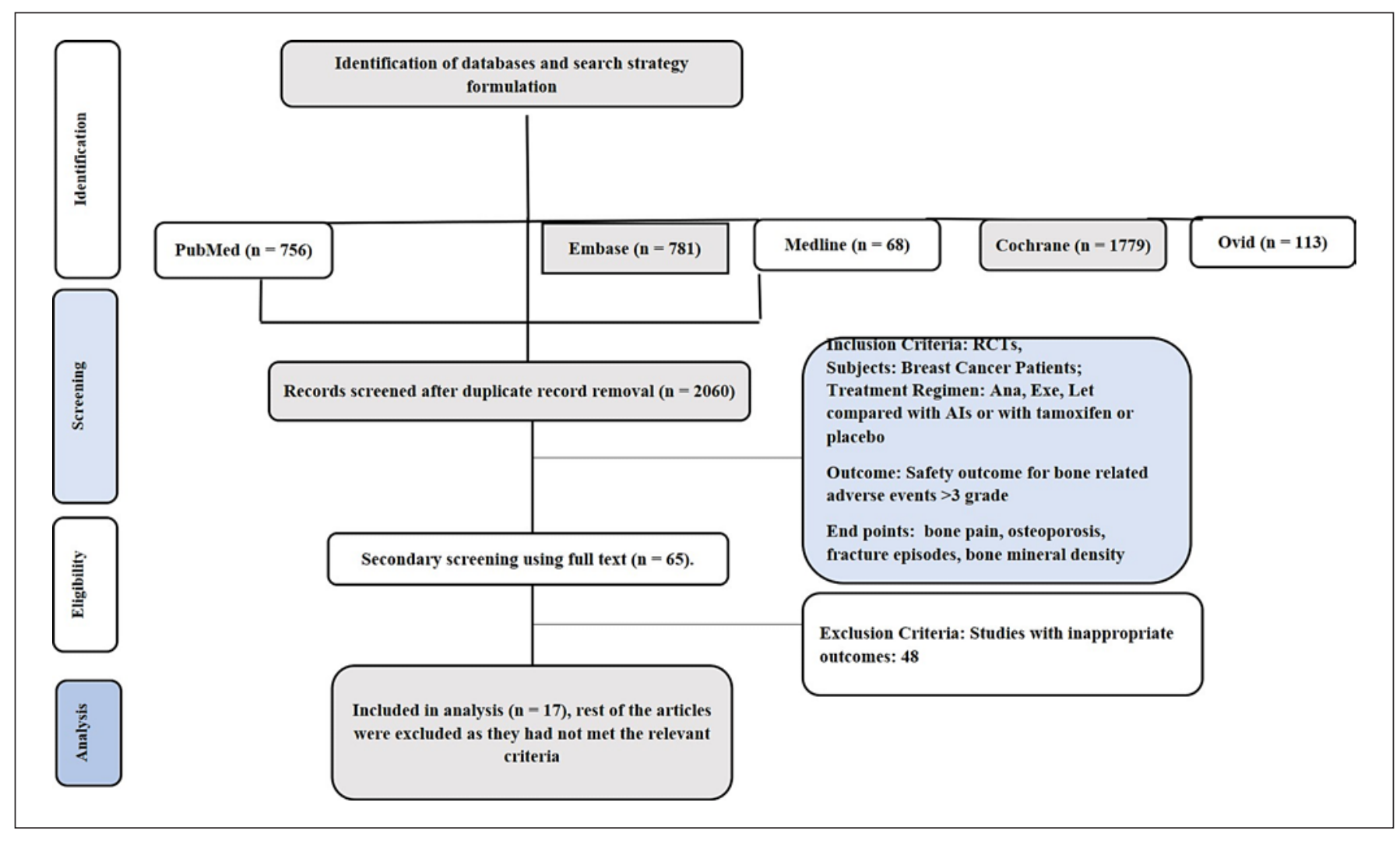

Fig. 1. Study selection flow diagram.

Publication Bias and Methodological Quality Assessment

Publication bias for bone safety outcomes was assessed by visual inspection of comparison-adjusted funnel plots. Methodological quality assessment of the included studies was assessed by the Cochrane risk-of-bias tool.

\section{Outcomes and Endpoints}

At the data extraction stage, all bone-related endpoints were included for extraction. Endpoints included were incidences of bone pain, osteoporosis, fracture episodes, and changes in bone mineral density (BMD) in the hip and lumbar spine. The final analysis included only $\geq$ grade 3 AEs.

\section{Network Geometry}

For arriving at a proper network geometry, published randomized trials were analyzed. Evidence from the systematic review was sorted based on the intervention and comparator drugs for each endpoint. Each distinct drug was considered as a node in network, and connecting effect estimates were considered as edges. Disconnected nodes were connected with indirect evidence to complete the network for all endpoints. Publication-bias funnel plot was represented by an endpoint with maximum direct evidence as revealed by the number of studies. There were 5 expected nodes (tamoxifen, placebo, exemestane, letrozole, and anastrozole), and the overall network geometry was established considering all endpoints. Accordingly, direct evidence for any of the study endpoints was used to connect the nodes to establish the network geometry. Endpoints with a steroidal node and a minimum of 1 nonsteroidal node and/or tamoxifen were included.

\section{Statistical Analysis}

Data management, relevancy, duplication removal, and assessment of eligibility were performed using Microsoft Excel. Armlevel data with respect to the different bone safety events were extracted and used. The statistical software "R" was used. The Gemtc package based on generalized linear modeling was used for random Bayesian analysis [27]. Model heterogeneity was assessed using the $I^{2}$ statistic. Model fitting was performed using convergence between prior and posterior values using deviance information criteria (DIC). Random and fixed effects were selected based on the DIC and $I^{2}$ values. The model with the lower DIC and $I^{2}$ values was used for the analysis. The odds ratio (OR) was the effect estimate used for the comparison of all endpoints, except changes in $\mathrm{BMD}$. The mean reduction from baseline was the effect estimate used for the analysis of BMD. Effect estimates from indirect pairwise comparison of exemestane with other 2 AIs and surface under the cumulative ranking curve (SUCRA) values were used to interpret results. Most studies reported statistically nonsignificant effect estimates for bone safety events, and SUCRA values were used for identifying the treatment arm that reduced the incidence of bone safety events as compared with tamoxifen and placebo.

\section{Results}

\section{Characteristics of Eligible Articles}

A total of 2,090 consolidated studies were found, which were further curated to exclude duplicates and irrelevant articles using title and abstract during preliminary screening. A study flow diagram with results of the search strat- 


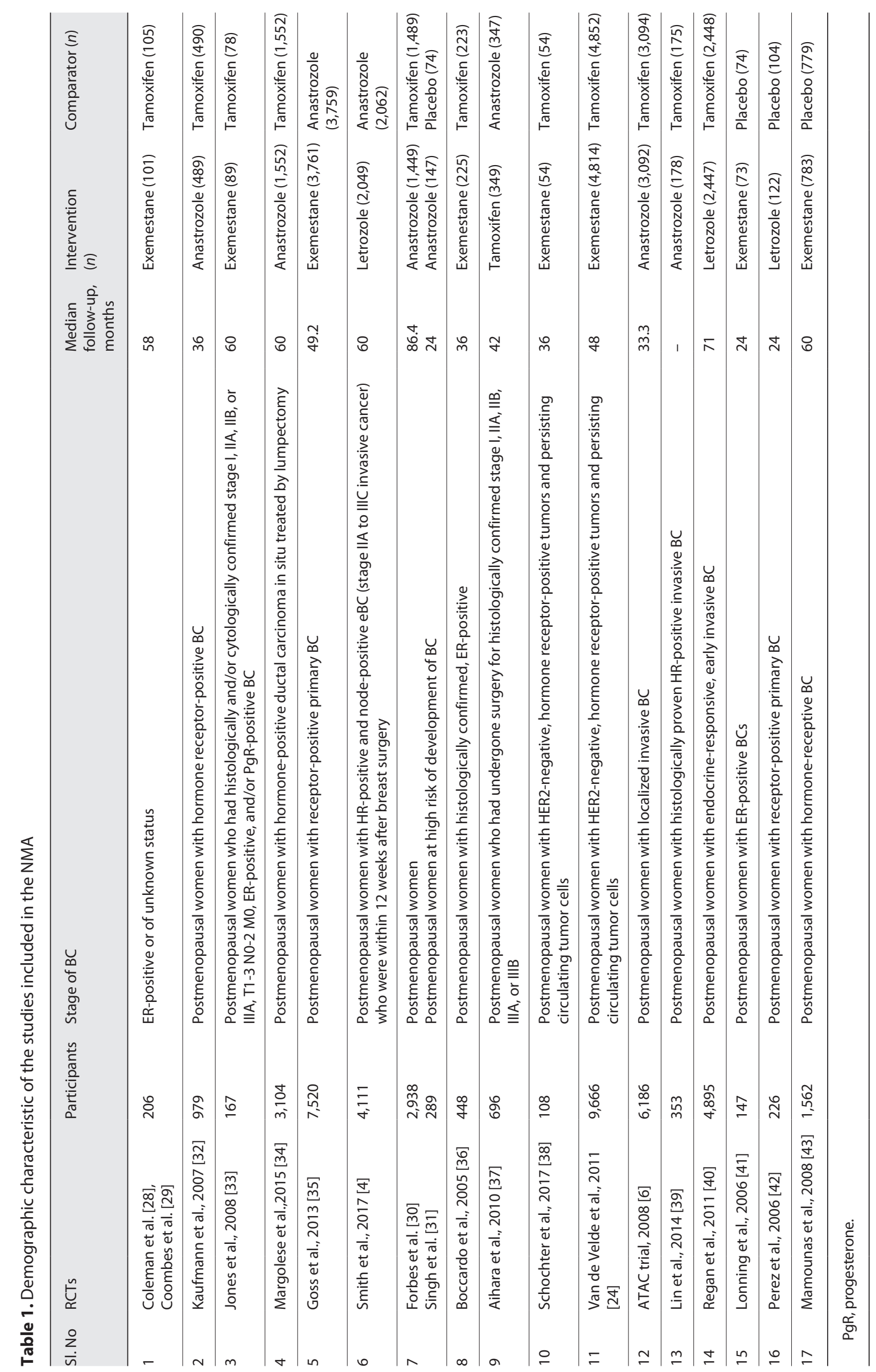



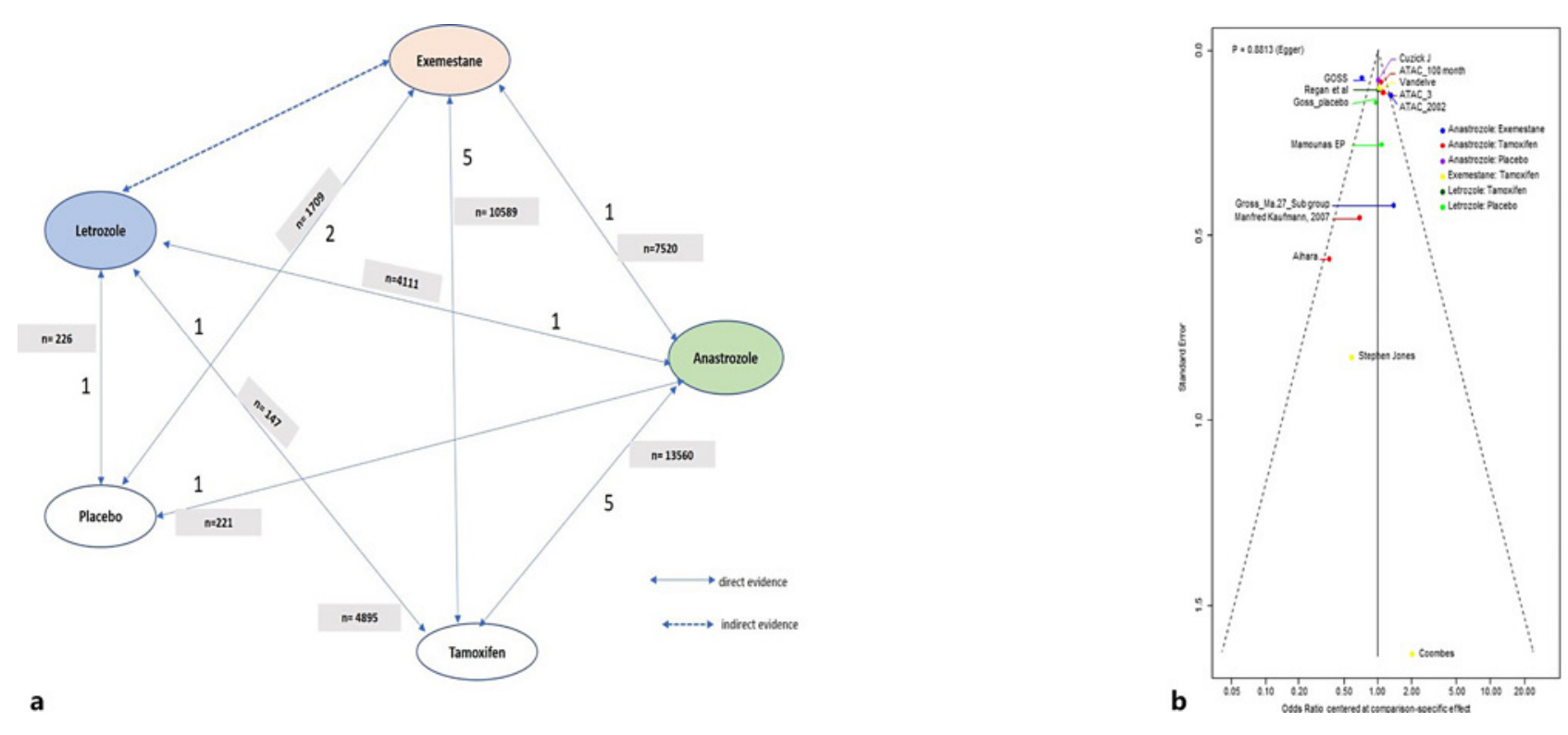

Fig. 2. a Network diagram of evidences to show the number of studies obtained as direct evidences. b Comparison of adjusted funnel plot for bone-related outcomes.

egy as per the PRISMA guidelines is shown in Figure 1. After the initial screening, 17 distinct studies were further scrutinized and considered eligible. Coleman et al. [28] and Coombes et al. [29] reported data from the same trial, and both studies were used. Forbes et al. [30] and Singh et al. [31] reported data from the same trial with a placebo-controlled study as a sub-study. Relevant data from the outcomes were extracted. The baseline demographics of studies are provided in Table 1. A total of 43,708 patients were analyzed for NMA.

\section{Network Geometry}

Network geometry differed based on the endpoint assessed. The most frequently reported direct comparisons were anastrozole and tamoxifen (5 studies), tamoxifen and exemestane (5 studies), and placebo (3 studies). Endpoints assessed were fracture episodes, osteoporosis, bone pain, and changes in BMD. Myalgia was excluded for analysis as there were no studies reporting the incidence of myalgia in patients treated with exemestane. Network geometry consists of 5 nodes and 6 edges for fracture, osteoporosis, bone pain, respectively. The overall network is shown in Figure 2a.

\section{Publication Bias}

Publication bias assessed by visual inspection of treatment-adjusted funnel plots and Egger's regression revealed negligible publication bias for bone-related outcomes (shown in Fig. 2b).

\section{Bone Pain}

There were 7 studies that reported bone pain-related events associated with AI therapy, of which tamoxifen was the comparator for 5 studies, and placebo was the comparator for 2 studies. On the basis of heterogeneity, the data fit was better with the random effects model (DIC: $30.08, I^{2}: 8 \%$ ); hence, it was used for analysis. Compared with tamoxifen, all AIs had higher odds for bone pain (shown in Fig. 3a). With placebo-controlled studies, odds of bone pain incidences were lesser (shown in Fig. 3b). Among AIs, exemestane had lower odds for bone pain than anastrozole (exemestane vs. anastrozole) (OR: $0.6376,95 \%$ credibility interval [CrI]: $0.0964-2.587)$ and letrozole (OR: 0.5496, 95\% CrI: 0.0264-7.3337; Table 2). ORs revealed lack of statistical significance for all comparisons. According to cumulative rank plots (shown in Fig. 3c) and SUCRA values as outlined in Table 2, exemestane was the best among the AIs followed by anastrozole and letrozole (the ranking of SUCRA is as follows: exemestane: 0.595 , anastrozole: 0.3525 , and letrozole: 0.3395).

\section{Osteoporosis}

Ten studies reported osteoporosis-related events associated with AI therapy. One trial (MA 17.R) [35] reported data with BMD after a follow-up with placebo for 5 years. On the basis of heterogeneity, the data fit was better with the random effects model (DIC: $38.71, I^{2}: 0 \%$ ); hence, it was used for analysis. All the AIs had significantly higher odds of osteoporosis (shown in Fig. 4a) than tamoxifen. 


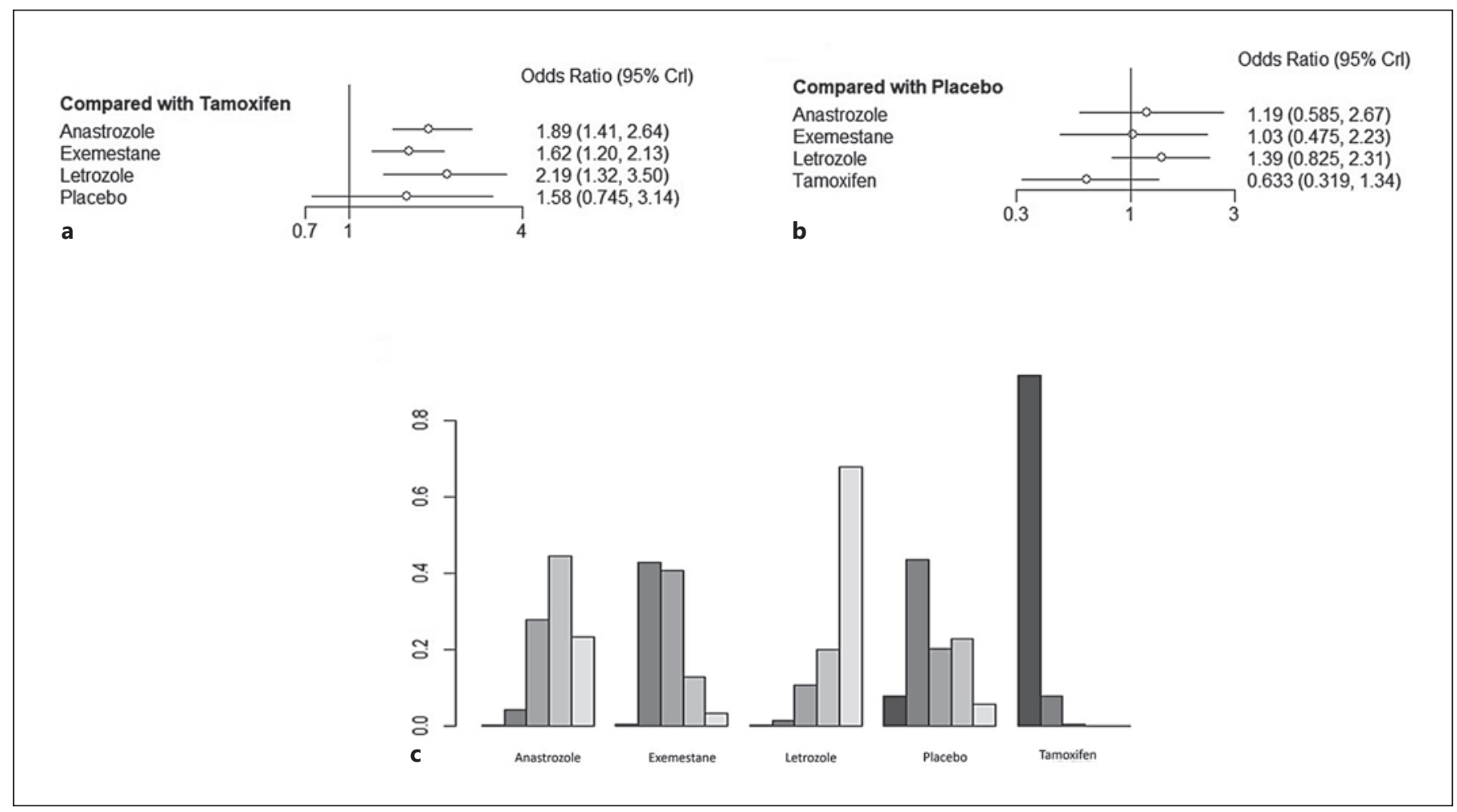

Fig. 3. a Forest plot depicting relative safety with respect to bone pain with tamoxifen as comparator. b Forest plot depicting relative safety with respect to bone pain with placebo as comparator. c Cumulative rank plot for comparison of treatments based on safety for bone pain with tamoxifen and placebo as comparator.

Table 2. Effect estimates and rank probability of exemestane in comparison to other Als for bone-related events

\begin{tabular}{lllll}
\hline Events & $\begin{array}{l}\text { Studies, } \\
n\end{array}$ & $\begin{array}{l}\text { Exemestane versus } \\
\text { anastrozole }(\mathrm{OR}, 95 \% \mathrm{Crl})\end{array}$ & $\begin{array}{l}\text { Exemestane versus } \\
\text { letrozole }(\mathrm{OR}, 95 \% \mathrm{Crl})\end{array}$ & SUCRA values \\
\hline Bone pain & 7 & $0.6376(0.0964-2.587)$ & $0.5496(0.026-7.337)$ & $\begin{array}{l}\text { Exemestane: } 0.59 \\
\text { Anastrozole: } 0.32 \\
\text { Letrozole: } 0.33\end{array}$ \\
\hline Osteoporosis & 10 & $0.8594(0.57-1.16)$ & $0.7358(0.4301-1.307)$ & $\begin{array}{l}\text { Exemestane: } 0.56 \\
\text { Anastrozole: } 0.28 \\
\text { Letrozole: } 0.11\end{array}$ \\
\hline Fracture episodes & 10 & $0.8497(0.628-1.119)$ & $0.8051(0.4561-1.295)$ & $\begin{array}{l}\text { Exemestane: } 0.62 \\
\text { Anastrozole: } 0.25 \\
\text { Letrozole: } 0.18\end{array}$ \\
\hline
\end{tabular}

Among the AIs, exemestane had lower odds of osteoporosis than anastrozole (OR: 0.8594, 95\% CrI: 0.5766-1.168) and letrozole (OR: 0.7358, 95\% CrI: 0.4301-1.307; Table 2). The ORs revealed lack of statistical significance for all comparisons both with tamoxifen and placebo (shown in Fig. 4a, b). According to cumulative rank plots (shown in Fig. 4c) and SUCRA values, exemestane was the best among the AIs followed by anastrozole and letrozole (the ranking of SUCRA is as follows: exemestane: 0.56 , anastrozole: 0.28 , and letrozole: 0.11 ; Table 2).
Fracture Episodes

Ten studies reported fracture-related events associated with AI therapy, of which 7 distinct studies reported outcomes as compared with tamoxifen, whereas 3 distinct RCTs were placebo-controlled studies. On the basis of heterogeneity, the data fit was better with a random effects model (DIC: $52.08, I^{2}: 7 \%$ ); hence, it was used for analysis. Compared with tamoxifen, all the 3 AIs had higher odds of fracture episodes (shown in Fig. 5a) in placebo-controlled RCTs, and exemestane 


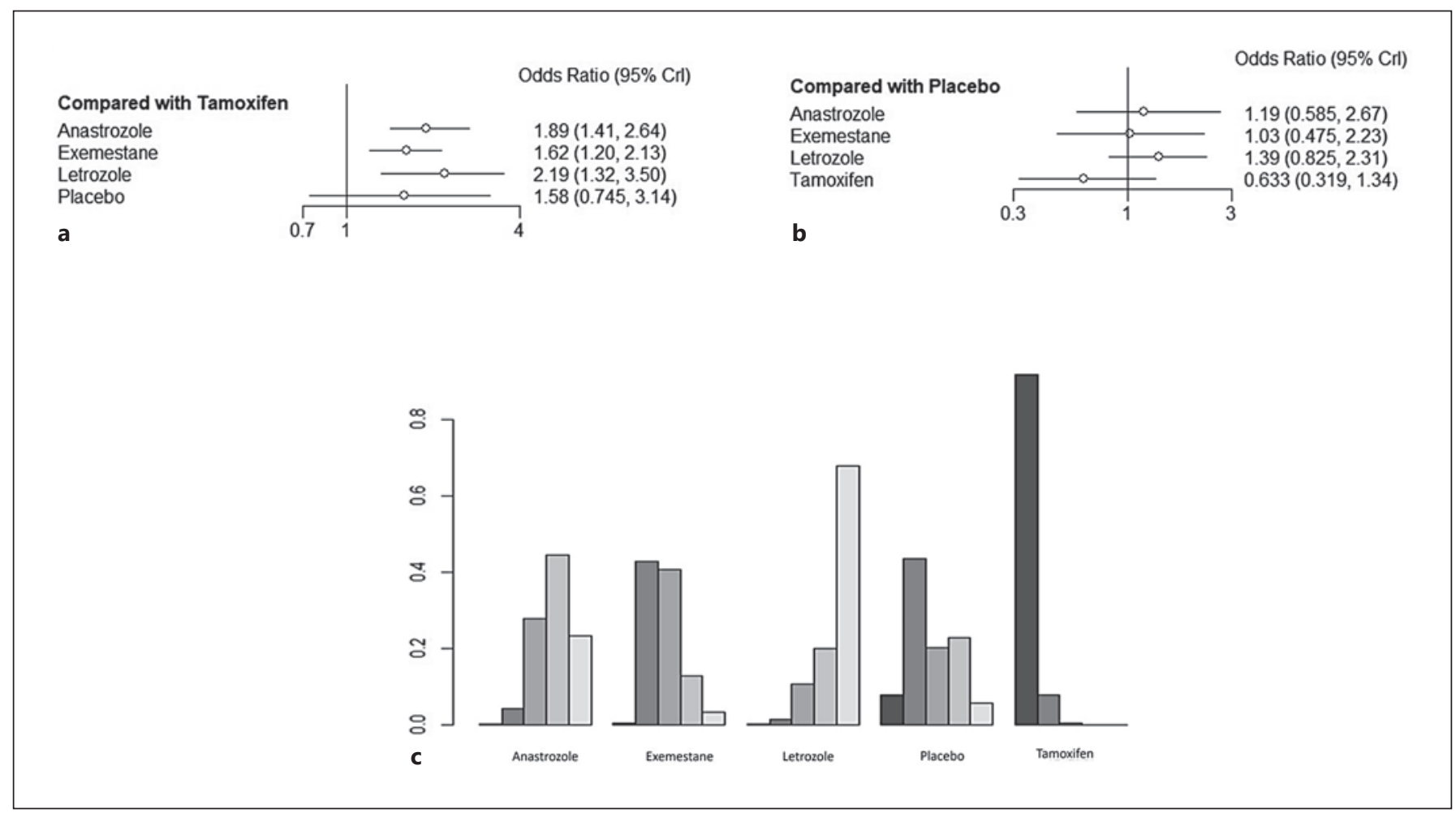

Fig. 4. a Forest plot depicting the relative safety with respect to osteoporosis with tamoxifen as comparator. b Forest plot depicting the relative safety with respect to osteoporosis with placebo as comparator. c Cumulative rank plot for comparison of treatments based on safety for osteoporosis with tamoxifen and placebo as comparators.

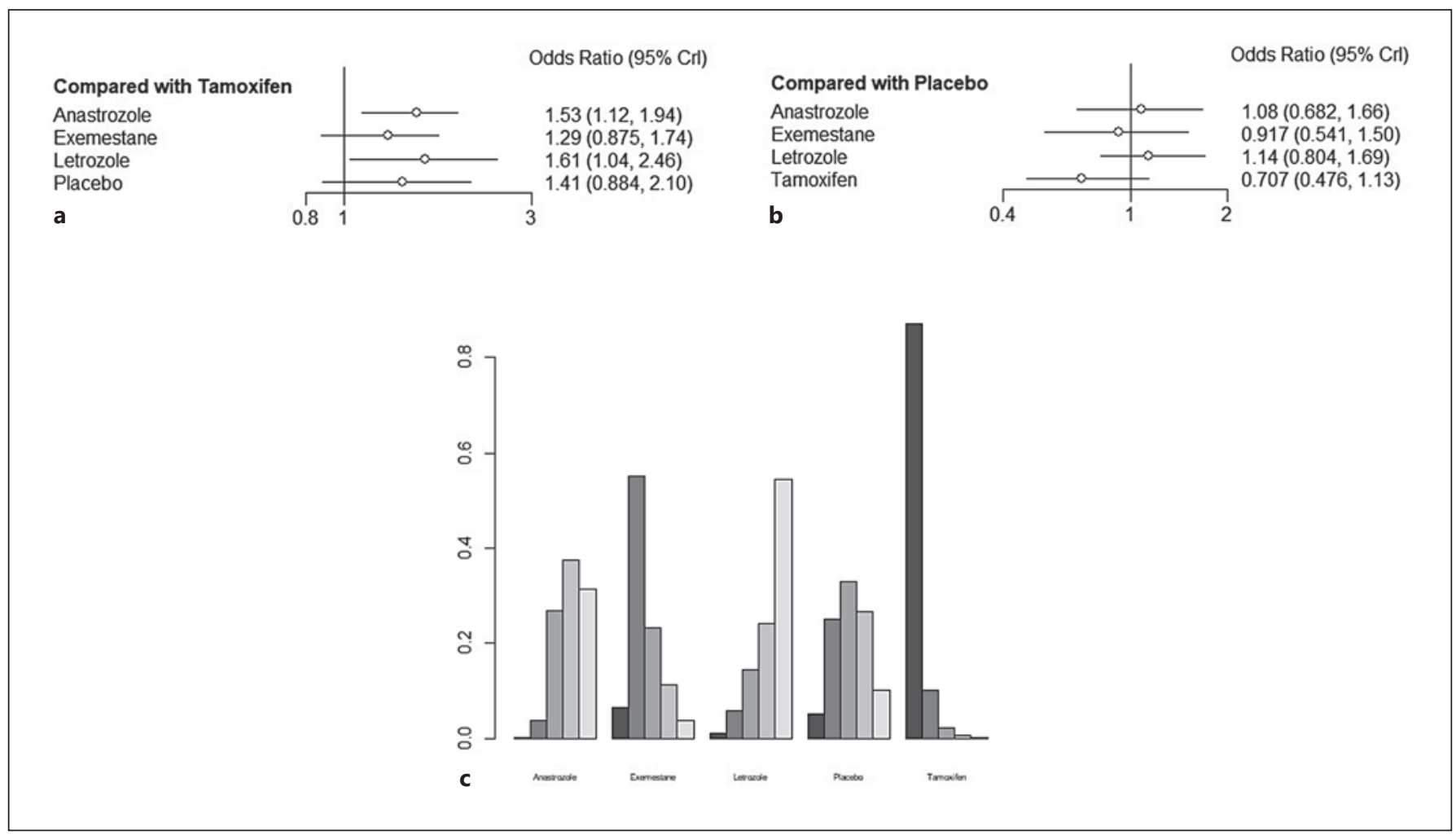

Fig. 5. a Forest plot depicting the relative safety with respect to fracture episodes as compared to tamoxifen. b Forest plot depicting the relative safety with respect to fracture episodes as compared to placebo. c Cumulative rank plot for comparison of treatments based on safety for fracture episodes. 


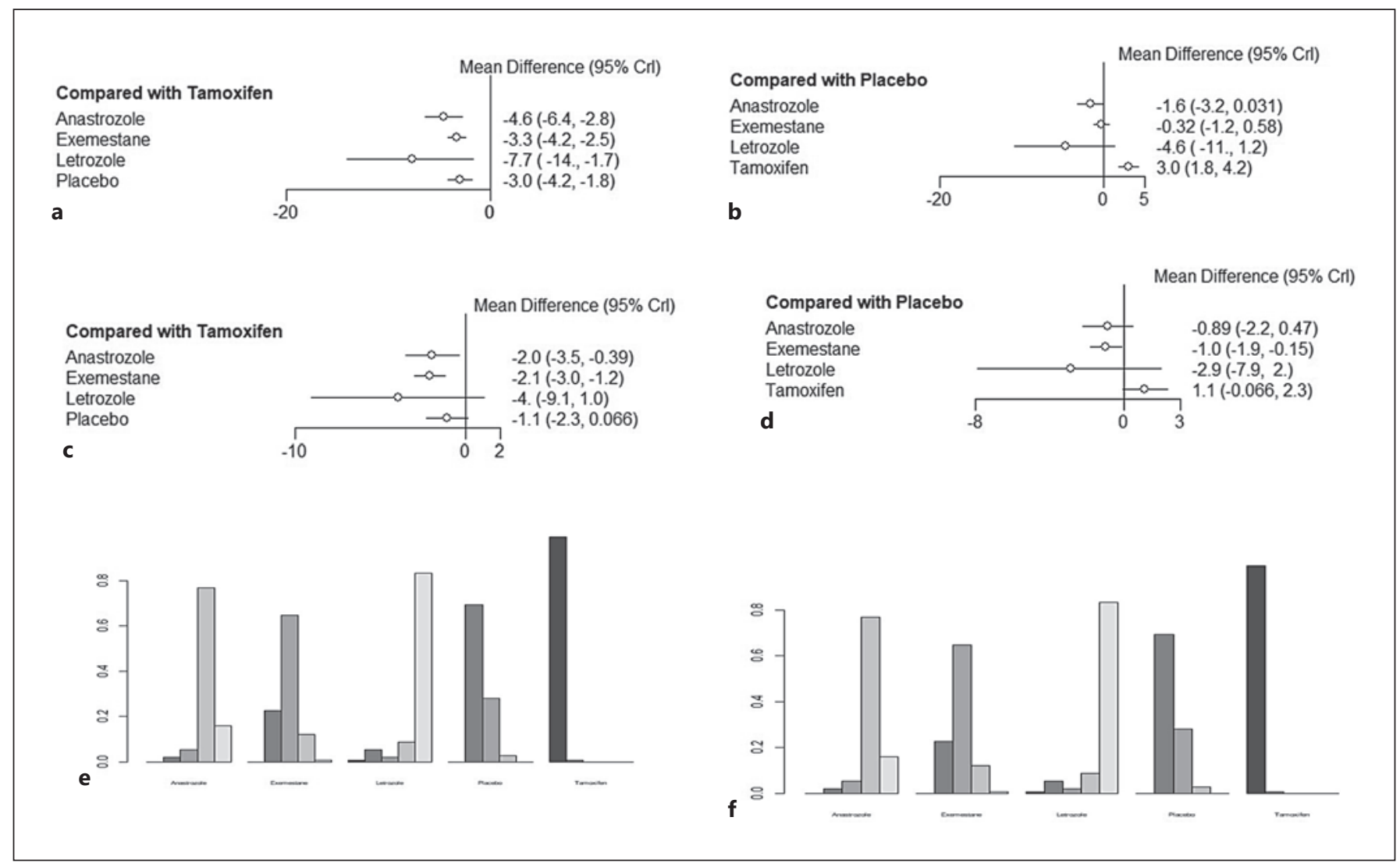

Fig. 6. a Forest plot depicting the reduction in BMD in the lumbar spine compared with tamoxifen. b Forest plot depicting the reduction in BMD in the lumbar spine compared with placebo. c Forest plot depicting the reduction in BMD in the hip compared with tamoxifen. $\mathbf{d}$ Forest plot depicting the reduction in BMD in the hip compared with placebo. e Cumulative rank plot for comparison of treatments based on BMD at hip. $\mathbf{f}$ Cumulative rank plot for comparison of treatments based on BMD at lumbar spine.

had lower incidences of fracture episodes as compared with the other 2 AIs (shown in Fig. 5b). Among the AIs, patients treated with exemestane had lower incidence of fracture episodes in comparison with anastrozole (OR: 0.8497, 95\% CrI: 0.628-1.119) and letrozole (OR: 0.8051, 95\% CrI: 0.4561-1.295). ORs revealed lack of statistical significance for all comparisons with both tamoxifen and placebo. According to cumulative rank plots (shown in Fig. 5c) and SUCRA values, exemestane was the best among the AIs followed by letrozole and anastrozole (the ranking of SUCRA is as follows: exemestane: 0.62 , anastrozole: 0.25 , and letrozole: 0.18 ; Table 2).

\section{Changes in BMD}

Eight studies reported changes in BMD in hip and lumbar spines, of which 5 studies compared BMD with tamoxifen, and 3 studies compared BMD with placebo. On the basis of heterogeneity, the data fit was better with a fixed-effects model (hip spine, DIC: $8.36, I^{2}: 0 \%$; lumbar spine, DIC: $\left.10.37 \%, I^{2}: 0 \%\right)$; hence, it was used for analysis. Treatment with both anastrozole and exemestane led to an increased reduction in BMD from baseline (shown in Fig. 6a, c) compared with tamoxifen. In placebo-controlled RCTs, all the 3 AIs showed a reduction in BMD from baseline in both hip and lumbar spine (shown in Fig. 6b, d). Compared with exemestane, a mean reduction in BMD from baseline was high in patients treated with anastrozole and letrozole in lumbar (anastrozole [mean reduction]: 1.252, 95\% CrI: -0.476 to 2.963 and letrozole [mean reduction]: 4.38 , 95\% CrI: -1.641 to 1.641 ) and hip (anastrozole [mean reduction: $-0.1419,95 \% \mathrm{CrI}:-1.558$ to -1.259 and letrozole [mean reduction]: $-1.819,95 \% \mathrm{CrI}:-6.854$ to -3.207). According to cumulative rank plots (shown in Fig. 6e, f) and SUCRA values, exemestane was better than letrozole for hip (hip: exemestane: 0.29 and letrozole: 0.16 and better than both anastrozole and letrozole in lumbar spine: exemestane: 0.52 , anastrozole: 0.23 , and letrozole: 0.07 ). 


\section{Discussion}

Of the 3 AIs, evidence from preclinical models and clinical studies suggests that exemestane is a good candidate for long-term treatment of early BC in the adjuvant setting owing to its antiestrogenic effects such as bone resorption and mild androgenic activity [15]. Of the 3 bone safety endpoints assessed, exemestane was better than letrozole and anastrozole as revealed by SUCRA values. Assessment of bone safety was not a primary endpoint in any of the included studies. Incidences reported in individual studies were not adequately powered in terms of statistical significance. Standard errors for bone safety events were relatively high in included studies that reflected in indirect effect estimates derived from Bayesian models. The SUCRA values suggested that exemestane was associated with lower incidences of bone fracture, bone pain, and osteoporosis. The mean reduction in $\mathrm{BMD}$ was lower in patients treated with exemestane than those treated with anastrozole, which could be the biological reason for the lower incidence of bone safety events in patients treated with exemestane.

Both nonsteroidal and steroidal AIs reduced contralateral primary BCs compared with tamoxifen [44]. The side effects of AIs differ from those of SERMs, reflecting the specific mechanism of action of these drugs [11]. There is strong evidence that AIs are well tolerated [45]. Owing to the difference in the mode of action of steroidal and nonsteroidal AIs, the incidences of safety events were also presumed to be different. In early preclinical studies, letrozole was found to achieve higher levels of wholebody aromatization than exemestane and anastrozole (>99.1\% for letrozole vs. $97.3 \%$ for anastrozole vs. $97.9 \%$ for exemestane) [35, 46, 47]. Hence, letrozole might lead to higher incidence of safety events. The results of NMA also suggest that letrozole was associated with a higher incidence of bone pain and osteoporosis.

BMD and bone turnover are more accurate predictors of bone safety events but are not reported for all the studies. Hence, BMD reported for exemestane, anastrozole, and letrozole was under analysis. The results suggested that lower reduction was reported in BMD in both hip and lumbar spine in patients treated with exemestane than those treated with anastrozole at 2 years. Reduction in BMD in patients treated with exemestane was reported to be less after 2 years suggesting that the effects of exemestane on BMD and bone safety might be better after prolonged treatment [48]. This suggests that exemestane is the best long-term adjuvant therapy.

All the 3 AIs have been associated with an increased risk of osteoporosis and/or fractures in adjuvant BC trials. These trials did not report vertebral fracture events and may have used different definitions for osteoporosis. Baseline $\mathrm{T}$ scores were not reported in the majority of studies, and randomization was also not stratified based on baseline $T$ scores. The sequence of endocrine therapy use might also influence bone safety events. In the majority of exemestane studies, patients were previously treated with tamoxifen; hence, the observed incidence of bonerelated events might also be due to the residual effect of tamoxifen. Other AIs have been evaluated in the first-line endocrine therapy settings. Despite that, results suggest that exemestane was associated with a lower incidence of bone safety events in our analysis.

All trials investigated the effects of AIs on bone-related safety events when compared with tamoxifen. The accurate reason for the difference in BMD observed between $\mathrm{AI}$ and tamoxifen would be difficult to decipher. Comparison of estimates with placebo as a comparator was done to determine the effect of AI on bone health.

The safety profile is important as a number of AEs would greatly influence the adherence rate to drugs [49]. Data on predefined AEs were usually collected to monitor the safety profile of anastrozole, exemestane, and letrozole as compared with that of tamoxifen [35]. Up to 30\% of all women taking AIs report myalgia or arthralgia [50]. In the adjuvant setting, the rate of musculoskeletal symptoms is significantly higher for all the third-generation AIs compared with tamoxifen [48]. These events generally emerge early in treatment and are of low grade and improve with time [35]. AIs cause an increase in both bone resorption and formation [35]. Osteoporosis and increased fracture rates occur in some patients when using AIs [35]. Although preclinical studies suggested that bone loss may be less during treatment with a steroidal AI (exemestane) compared with nonsteroidal AIs (anastrozole and letrozole), there is no clinical trials evidence confirming these hypotheses [35]. In contrast, RCT of healthy volunteers demonstrated that all the AIs have a similar effect on bone turnover [51]. As a result of early screening for osteopenia and osteoporosis whenever AIs are implemented in patients with early BCs and liberal use of calcium, vitamin $\mathrm{D}$, and bisphosphonates, the issue of bone loss seems to be solved for the majority of patients [50].

The results are in accordance with previous studies, which have reported that musculoskeletal symptoms and decreased BMD are the anticipated effects of hormonal therapies, such as AIs, that produce menopause-like effects [3]. In the MA.27 trial [15], arthralgia, muscle pain, and fractures were reported in both exemestane and anastrozole treatment groups; however, exemestane was associated with a significantly lower incidence of self-reported new-onset osteoporosis compared with anastrozole. Real-world studies show that in terms of bone loss, steroidal AI is expected to be superior to nonsteroidal AI because steroids in bone would be favorable for bone metabolism. In an observational study, exemestane treatment maintained BMD of femoral neck (FN), suggesting 
that exemestane is favorable for BMD of FN [52]. The reason why steroidal AI induces less damage to $\mathrm{FN}$ compared with nonsteroidal AI is unknown, but steroidal AI might affect bone turnover directly through its structure. Oesterreich et al. [53] reported that urine N-telopeptide concentration was significantly increased in patients treated with exemestane but not in those treated with letrozole[54]. Another observational study in a real-world setting, conducted with everolimus plus exemestane in postmenopausal women with hormone receptor-positive, HER2-negative advanced $\mathrm{BC}$ showed that this combination had lower incidents of bone-related events [52].

The advantages of NMA are that it not only increases statistical power by incorporating evidence from both direct (head-to-head) and indirect comparisons across all 4 interventions but also can provide insights into the relative effectiveness of interventions that have never been directly compared such as anastrozole therapy, letrozole therapy, and exemestane therapy. It combines direct and indirect evidence on the relative effectiveness of several interventions with respect to randomization. NMA with the Bayesian framework, at best, provides a robust and mathematically coherent framework and shows a good prediction accuracy even with rather small sample sizes [54].

One of the limitations of this analysis is that there were a limited number of studies for some of the endpoints but that would not affect the overall power of the study. Heterogeneity is difficult to avoid because the patient profile differed for each trial. The main strength of this analysis is the fact that this is the first NMA comparing comprehensive safety events of different AIs covering bone-related events.

To conclude, results of NMA suggest that the lower incidences of bone-related safety events were observed in patients treated with exemestane than those treated with letrozole and anastrozole. Furthermore, randomized controlled trials and prospective studies in a large cohort are warranted to determine the side effects of AIs on bone-related health in this patient population. The existence of a superior or patient-specific AI remains questionable. The decision to use one specific AI should be based on their respective safety profiles, maturity of data, and availability of results within chosen clinical strategies. As clinicians are now shifting more to AIs, it is imperative to have an evidence-based decision algorithm to make an informed choice. Bone-related safety events remain an important consideration for the increase in the number of incidents such as fracture and joint stiffness that lowers the quality of life and in turn affects adherence to the treatment regimen.

\section{Acknowledgments}

We would like to thank Pfizer Medical Affair, along with Dr. Amit Bhat of Indegene Pvt Ltd., India for their editorial and medical writing support. Also, we acknowledge Divya of Indegene Pvt Ltd., for statistical analysis support.

\section{Statement of Ethics}

An ethics statement is not applicable because this study is based exclusively on published literature.

\section{Conflict of Interest Statement}

The authors have no conflicts of interest to declare.

\section{Funding Sources}

No funding was received for this study.

\section{Author Contributions}

Substantial contributions to the conception or design of the work or the acquisition, analysis, or interpretation of data for the work: Shanshan Chen and Lan Bo. Drafting the work or revising it critically for important intellectual content: Lan Bo, Dan Lv, and Fei Ma. Final approval of the version to be published: Shanshan Chen, Lan Bo, Dan Lv, and Fei Ma. Agreement to be accountable for all aspects of the work in ensuring that questions related to the accuracy or integrity of any part of the work are appropriately investigated and resolved: Shanshan Chen, Lan Bo, Dan Lv, and Fei Ma.

\section{Data Availability Statement}

All data generated or analyzed during this study are included in this article. Further inquiries can be directed to the corresponding author.

\section{References}

1 Bray F, Ferlay J, Soerjomataram I, Siegel RL, Torre LA, Jemal A. Global cancer statistics 2018: GLOBOCAN estimates of incidence and mortality worldwide for 36 cancers in 185 countries. CA Cancer J Clin. 2018;68(6):394424 .
2 Lumachi F, Santeufemia DA, Basso SM. Current medical treatment of estrogen receptorpositive breast cancer. World J Biol Chem. 2015 Aug;6(3):231-9.

3 Tomao F, Spinelli G, Vici P, Pisanelli GC, Cascialli G, Frati L, et al. Current role and safety profile of aromatase inhibitors in early breast cancer. Expert Rev Anticancer Ther. 2011 Aug;11(8):1253-63. 
4 Smith IE, Dowsett M, Ebbs SR, Dixon JM, Skene A, Blohmer J-U, et al. Neoadjuvant treatment of postmenopausal breast cancer with anastrozole, tamoxifen, or both in combination: the Immediate Preoperative Anastrozole, Tamoxifen, or Combined with Tamoxifen (IMPACT) multicenter doubleblind randomized trial. J Clin Oncol. 2005 Aug;23(22):5108-16.

5 Cataliotti L, Buzdar AU, Noguchi S, Bines J, Takatsuka Y, Petrakova K, et al. Comparison of anastrozole versus tamoxifen as preoperative therapy in postmenopausal women with hormone receptor-positive breast cancer: the pre-operative "arimidex" compared to tamoxifen (PROACT) trial. Cancer. 2006 May;106(10):2095-103.

6 Arimidex, Tamoxifen, Alone or in Combination (ATAC) Trialists' Group, Forbes JF, Cuzick J, Buzdar A, Howell A, Tobias JS, et al. Effect of anastrozole and tamoxifen as adjuvant treatment for early-stage breast cancer: 100-month analysis of the ATAC trial. Lancet Oncol. 2008 Jan;9(1):45-53.

7 Thürlimann B, Hess D, Köberle D, Senn I, Ballabeni P, Pagani O, et al. Anastrozole ("Arimidex") versus tamoxifen as first-line therapy in postmenopausal women with advanced breast cancer: results of the doubleblind cross-over SAKK trial 21/95: a substudy of the TARGET (tamoxifen or "arimidex" randomized group efficacy and tolerability) trial. Breast Cancer Res Treat. 2004 Jun;85(3):247-54.

8 Goetz MP, Gradishar WJ, Anderson BO, Abraham J, Aft R, Allison KH, et al. NCCN guidelines insights: breast cancer, version 3.2018 - featured updates to the NCCN guidelines. J Natl Compr Canc Netw. 2019 Feb;17(2):118-26.

9 ESMO. ESMO clinical practice guidelines: breast cancer [Internet]. [cited 2020 May 8]. Available from: https://www.esmo.org/ guidelines/breast-cancer.

10 Kwan ML, Yao S, Laurent CA, Roh JM, Quesenberry CP, Kushi LH, et al. Changes in bone mineral density in women with breast cancer receiving aromatase inhibitor therapy. Breast Cancer Res Treat. 2018 Apr;168(2):523-30.

11 Nabholtz JM. Long-term safety of aromatase inhibitors in the treatment of breast cancer. Ther Clin Risk Manag. 2008 Feb;4(1):189204.

12 Ramchand SK, Cheung YM, Yeo B, Grossmann $M$. The effects of adjuvant endocrine therapy on bone health in women with breast cancer. J Endocrinol. 2019 Jun;241(3):R111-24.

13 Simpson E, Santen RJ. Celebrating 75 years of oestradiol. J Mol Endocrinol. 2015 Dec;55(3): T1-20.

14 Evans CT, Ledesma DB, Schulz TZ, Simpson ER, Mendelson CR. Isolation and characterization of a complementary DNA specific for human aromatase-system cytochrome P-450 mRNA. Proc Natl Acad Sci U S A. 1986 Sep; 83(17):6387-91.

15 Goss PE, Hadji P, Subar M, Abreu P, Thomsen T, Banke-Bochita J. Effects of steroidal and nonsteroidal aromatase inhibitors on markers of bone turnover in healthy postmenopausal women. Breast Cancer Res. 2007 Aug;9(4):R52.
16 Paridaens R, Dirix L, Lohrisch C, Beex L, Nooij M, Cameron D, et al. Mature results of a randomized phase II multicenter study of exemestane versus tamoxifen as first-line hormone therapy for postmenopausal women with metastatic breast cancer. Ann Oncol. 2003 Sep;14(9):1391-8.

17 Amir E, Seruga B, Niraula S, Carlsson L, Ocaña A. Toxicity of adjuvant endocrine therapy in postmenopausal breast cancer patients: a systematic review and meta-analysis. J Natl Cancer Inst. 2011 Sep;103(17):1299-309.

18 Pisani P, Renna MD, Conversano F, Casciaro E, Di Paola M, Quarta E, et al. Major osteoporotic fragility fractures: risk factor updates and societal impact. World J Orthop. 2016; $7(3): 171$.

19 Zaccheo T, Giudici D, Lombardi P, di Salle E. A new irreversible aromatase inhibitor, 6-methylenandrosta-1,4-diene-3,17-dione (FCE 24304): antitumor activity and endocrine effects in rats with DMBA-induced mammary tumors. Cancer Chemother Pharmacol. 1989;23(1):47-50.

20 Goss PE, Tye LM. Anastrozole: a new selective nonsteroidal aromatase inhibitor. Oncology. 1997 Nov;11(11):1697-703; discussion 1707-8.

21 Goss PE, Smith RE. Letrozole for the management of breast cancer. Expert Rev Anticancer Ther. 2002 Jun;2(3):249-60.

22 Walker G, Xenophontos M, Chen L, Cheung K. Long-term efficacy and safety of exemestane in the treatment of breast cancer. Patient Prefer Adherence. 2013 Mar;7:245-58.

23 Bliss JM, Kilburn LS, Coleman RE, Forbes JF, Coates AS, Jones SE, et al. Disease-related outcomes with long-term follow-up: an updated analysis of the intergroup exemestane study. J Clin Oncol. 2012 Mar;30(7):709-17.

24 van de Velde CJH, Rea D, Seynaeve C, Putter $\mathrm{H}$, Hasenburg A, Vannetzel J-M, et al. Adjuvant tamoxifen and exemestane in early breast cancer (TEAM): a randomised phase 3 trial. Lancet. 2011 Jan;377(9762):321-31.

25 Aydiner A. Meta-analysis of breast cancer outcome and toxicity in adjuvant trials of aromatase inhibitors in postmenopausal women. Breast. 2013 Apr;22(2):121-9.

26 Hutton B, Salanti G, Caldwell DM, Chaimani A, Schmid CH, Cameron C, et al. The PRISMA extension statement for reporting of systematic reviews incorporating network metaanalyses of health care interventions: checklist and explanations. Ann Intern Med. 2015 Jun;162(11):777.

27 R package: Gemtc. [cited 2020 Jul 5]. Available from: http://cran.r-project.org/web/ packages/gemtc/index.html.

28 Coleman RE, Banks LM, Girgis SI, Kilburn LS, Vrdoljak E, Fox J, et al. Skeletal effects of exemestane on bone-mineral density, bone biomarkers, and fracture incidence in postmenopausal women with early breast cancer participating in the Intergroup Exemestane Study (IES): a randomised controlled study. Lancet Oncol. 2007 Feb;8(2):119-27.

29 Coombes R, Kilburn L, Snowdon C, Paridaens R, Coleman R, Jones S, et al. Survival and safety of exemestane versus tamoxifen after 2-3 years' tamoxifen treatment (Intergroup Exemestane Study): a randomised controlled trial. Lancet. 2007 Feb;369(9561):559-70.
30 Forbes JF, Sestak I, Howell A, Bonanni B, Bundred N, Levy C, et al. Anastrozole versus tamoxifen for the prevention of locoregional and contralateral breast cancer in postmenopausal women with locally excised ductal carcinoma in situ (IBIS-II DCIS): a double-blind, randomised controlled trial. Lancet. 2016 Feb;387(10021):866-73.

31 Singh S, Cuzick J, Mesher D, Richmond B, Howell A. Effect of baseline serum vitamin D levels on aromatase inhibitors induced musculoskeletal symptoms: results from the IBISII, chemoprevention study using anastrozole. Breast Cancer Res Treat. 2012 Apr;132(2): 625-9.

32 Kaufmann M, Jonat W, Hilfrich J, Eidtmann H, Gademann G, Zuna I, et al. Improved overall survival in postmenopausal women with early breast cancer after anastrozole initiated after treatment with tamoxifen compared with continued tamoxifen: the ARNO 95 Study. J Clin Oncol. 2007 Jul;25(19):2664-70.

33 Jones S, Stokoe C, Sborov M, Braun M, Ethirajan S, Kutteh L, et al. The effect of tamoxifen or exemestane on bone mineral density during the first 2 years of adjuvant treatment of postmenopausal women with early breast cancer. Clin Breast Cancer. 2008 Dec;8(6): 527-32.

34 Margolese RG, Cecchini RS, Julian TB, Ganz PA, Costantino JP, Vallow LA, et al. Anastrozole versus tamoxifen in postmenopausal women with ductal carcinoma in situ undergoing lumpectomy plus radiotherapy (NSABP B-35): a randomised, double-blind, phase 3 clinical trial. Lancet. 2016 Feb;387(10021): 849-56.

35 Goss PE, Hershman DL, Cheung AM, Ingle JN, Khosla S, Stearns V, et al. Effects of adjuvant exemestane versus anastrozole on bone mineral density for women with early breast cancer (MA.27B): a companion analysis of a randomised controlled trial. Lancet Oncol. 2014 Apr;15(4):474-82.

36 Boccardo F, Rubagotti A, Puntoni M, Guglielmini P, Amoroso D, Fini A, et al. Switching to anastrozole versus continued tamoxifen treatment of early breast cancer: preliminary results of the Italian Tamoxifen Anastrozole Trial. J Clin Oncol. 2005 Aug;23(22):5138-47.

37 Aihara T, Takatsuka Y, Ohsumi S, Aogi K, Hozumi Y, Imoto S, et al. Phase III randomized adjuvant study of tamoxifen alone versus sequential tamoxifen and anastrozole in Japanese postmenopausal women with hormoneresponsive breast cancer: N-SAS BC03 study. Breast Cancer Res Treat. 2010 Jun;121(2): 379-87.

38 Schochter F, Rack B, Tzschaschel M, Polasik A, Andergassen U, Trapp E, et al. Endocrine treatment with 2 years of tamoxifen versus 2 years of exemestane in postmenopausal patients with high-risk early breast cancer and persisting circulating tumor cells - First results of the SUCCESS C Endocrine Treatment Sub-Study. Oncol Res Treat. 2018;41(3):93-8.

39 Lin Y, Liu J, Zhang X, Li L, Hu R, Liu J, et al. A prospective, randomized study on hepatotoxicity of anastrozole compared with tamoxifen in women with breast cancer. Cancer Sci. 2014;105(9):1182-8. https://doi.org/10.1111/ cas. 12474 . 
40 Regan MM, Price KN, Giobbie-Hurder A, Thürlimann B, Gelber RD, International Breast Cancer Study Group and BIG 1-98 Collaborative Group. Interpreting breast international group (BIG) 1-98: a randomized, double-blind, phase III trial comparing letrozole and tamoxifen as adjuvant endocrine therapy for postmenopausal women with hormone receptor-positive, early breast cancer. Breast Cancer Res. 2011;13(3):209. https: //doi.org/10.1186/bcr2837.

41 Lønning PE, Geisler J, Krag LE, Erikstein B, Bremnes Y, Hagen AI, et al. Effects of exemestane administered for 2 years versus placebo on bone mineral density, bone biomarkers, and plasma lipids in patients with surgically resected early breast cancer. J Clin Oncol. 2005; 23(22): 5126-37. https: //doi. org/10.1200/JCO.2005.07.097.

42 Perez EA, Josse RG, Pritchard KI, Ingle JN, Martino S, Findlay BP, et al. Effect of letrozole versus placebo on bone mineral density in women with primary breast cancer completing 5 or more years of adjuvant tamoxifen: a companion study to NCIC CTG MA.17. J Clin Oncol. 2006;24(22):3629-35. https://doi. org/10.1200/JCO.2005.05.4882.

43 Mamounas EP, Jeong J-H, Wickerham DL, Smith RE, Ganz PA, Land SR, et al. Benefit from exemestane as extended adjuvant therapy after 5 years of adjuvant tamoxifen: intention-to-treat analysis of the National Surgical Adjuvant Breast And Bowel Project B-33 trial
J Clin Oncol. 2008;26(12):1965-71. https:// doi.org/10.1200/JCO.2007.14.0228.

44 Fabian CJ. The what, why and how of aromatase inhibitors: hormonal agents for treatment and prevention of breast cancer: hormonal agents for treatment and prevention of breast cancer. Int J Clin Pract. 2007 Sep; 61(12):2051-63.

45 Geisler J, Haynes B, Anker G, Dowsett M, Lønning PE. Influence of letrozole and anastrozole on total body aromatization and plasma estrogen levels in postmenopausal breast cancer patients evaluated in a randomized, cross-over study. J Clin Oncol. 2002 Feb;20(3):751-7.

46 Geisler J, King N, Anker G, Ornati G, Di Salle E, Lønning PE, et al. In vivo inhibition of aromatization by exemestane, a novel irreversible aromatase inhibitor, in postmenopausal breast cancer patients. Clin Cancer Res. 1998 Sep;4(9):2089-93.

47 Geisler J, King N, Dowsett M, Ottestad L, Lundgren S, Walton P, et al. Influence of anastrozole (arimidex), a selective, non-steroidal aromatase inhibitor, on in vivo aromatisation and plasma oestrogen levels in postmenopausal women with breast cancer. Br J Cancer. 1996 Oct;74(8):1286-91.

48 Partridge AH, Wang PS, Winer EP, Avorn J. Nonadherence to adjuvant tamoxifen therapy in women with primary breast cancer. J Clin Oncol. 2003 Feb;21(4):602-6.

49 Geisler J, Suzuki T, Helle H, Miki Y, Nagasaki S, Duong NK, et al. Breast cancer aromatase expression evaluated by the novel antibody 677: correlations to intra-tumor estrogen levels and hormone receptor status. J Steroid Biochem Mol Biol. 2010 Feb;118(4-5):237-41.

50 Eastell R, Adams J, Clack G, Howell A, Cuzick J, Mackey J, et al. Long-term effects of anastrozole on bone mineral density: 7-year results from the ATAC trial. Ann Oncol. 2011 Apr;22(4):857-62.

51 Ottestad L, Fronth L, Rajendiran S, Hege Aksnes L, Eikesdal HP, Støre Blix E, et al. Observational study of everolimus plus exemestane in postmenopausal women with hormone receptor-positive, HER2-negative advanced breast cancer. Acta Oncol. 2019 Mar;58(3): 385-7.

52 Hirano A, Inoue $\mathrm{H}$, Ogura $\mathrm{K}$, Hattori A, Yukawa $\mathrm{H}$, Sakaguchi S, et al. Long-term effect of exemestane therapy on bone mineral density supported by bisphosphonates: results of 5-year adjuvant treatment in postmenopausal women with early-stage breast cancer. Asia Pac J Clin Oncol. 2018 Oct;14(5):e238-42.

53 Oesterreich S, Henry NL, Kidwell KM, Van Poznak CH, Skaar TC, Dantzer J, et al. Associations between genetic variants and the effect of letrozole and exemestane on bone mass and bone turnover. Breast Cancer Res Treat. 2015 Nov;154(2):263-73.

54 Uusitalo L. Advantages and challenges of Bayesian networks in environmental modelling. Ecol Model. 2007 May;203(3-4):312-8. http://dx.doi. org/10.1016/j.ecolmodel.2006.11.033. 